\title{
Percepción de la Relación Parental entre Adolescentes Mayores y Menores de 15 Años
}

\author{
RAMÓN FLORENZANO U. ${ }^{1,2,4,5}$, MACARENA VALDÉS C. ${ }^{6}$, EUGENIO CÁCERES C. ${ }^{7}$, \\ MARTÍN CASASSUS R. ${ }^{8}$, ANGÉLICA SANDOVAL I. ${ }^{5}$, SYLVIA SANTANDER R. ${ }^{3}$, SILVIA CALDERÓN S. ${ }^{9}$
}

1. Instituto de Ciencias de la Familia, Universidad de los Andes.

2. Facultad de Medicina, Universidad de Chile.

3. Dirección de Investigación de la Universidad Metropolitana de Ciencias de la Educación.

4. Servicio de Psiquiatría del Hospital del Salvador.

5. Facultad de Psicología, Universidad del Desarrollo.

6. Enfermera Universitaria, Master en Salud Pública, School of Public Health, Chapel Hill, North Carolina, USA. Facultad de Medicina, Universidad de Chile.

7. Arquitecto, Master en Matrimonio y Familia, Universidad de Navarra, España. Instituto de Ciencias de la Familia, Universidad de los Andes.

8. Psicólogo. Servicio de Psiquiatría del Hospital del Salvador. Facultad de Psicología, Universidad del Desarrollo.

9. Enfermera Universitaria, Magister en Salud Pública, Facultad de Medicina, Escuela de Salud Pública, Universidad de Chile.

\begin{abstract}
The Perception of Parental Relationship: A Comparison of Adolescents Older and Younger Than 15 Years of Age

This study evaluates the difference in risk behavior and depressive symptomalogy in adolescents, as parenting styles change through developmental stages. A group of students older and younger than 15 years old, in Santiago's Metropolitan Region were screened for these behaviors and symptoms, focusing upon the relationship with parents. Barber's Cross National Adolescent Program test, adapted and validated in Chile, was given to a 1447 students, randomly selected in 14 Middle Schools. Barber's model was used to compare upbringing styles. Results show that parental support correlates with children's social initiative, psychological control shows positive correlation with depressive symptomatology, and behavioral control shows a negative correlation with antisocial behavior. The only variable showing age bracket variability is antisocial adolescent behavior, when external and internal aggressive behavior are compared. The results suggest that Barber's model can be applied among Chilean adolescents 10 to 19 years of age. The implications of these findings, and possible preventive actions are presented.

(Key words: Adolescence, family, pharmacodependency).

Rev Chil Pediatr 2009; 80 (6): 520-527
\end{abstract}

Trabajo recibido el 31 de marzo de 2009, devuelto para corregir el 18 de mayo de 2009, segunda versión el 10 de agosto de 2009, aceptado para publicación el 17 de septiembre de 2009.

Investigación realizada con un subsidio a la investigación de la Universidad de los Andes (Proyecto FAI MED 003-07: Depresión, violencia y suicidio adolescentes: el rol de la familia en poblaciones clínicas y no clínicas).

Correspondencia a:

Dr. Ramón Florenzano U.

E-mail: rflorenzano@gmail.com 


\section{RESUMEN}

Este trabajo estudió la variación de las conductas de riesgo y sintomatología depresiva en adolescentes, según los cambios de estilos de crianza parentales en las distintas etapas del desarrollo. En él, se describe la frecuencia de esas conductas y síntomas en una muestra representativa de estudiantes de la Región Metropolitana de Santiago de Chile, con foco en las relaciones con sus padres, y en cómo éstos enfrentan la crianza en los hijos mayores o menores de 15 años. Se aplicó el instrumento CNAP (Cross National Adolescent Program), de Barber y cols, adaptado y validado para Chile por éste equipo de investigadores. En éste trabajo se analizan resultados de una primera aplicación a 1447 estudiantes de 14 establecimientos de enseñanza media aleatoriamente seleccionados. Se utiliza el modelo explicativo de Barber para comparar los estilos parentales diferenciales de crianza, encontrando que el apoyo parental se correlaciona positivamente con la iniciativa social de los hijos, que el control psicológico se correlaciona positivamente con síntomas depresivos y el control conductual lo hace negativamente con la conducta antisocial. Sólo el comportamiento antisocial adolescente muestra diferencias por grupo de edad al comparar conductas autoagresivas y heteroagresivas. Se concluye que el modelo de Barber puede aplicarse a la realidad chilena a adolescentes entre de 10 a 19 años y se analizan las implicancias de estos hallazgos para posibles intervenciones preventivas.

(Palabras clave: Adolescencia, familia, farmacodependencia).

Rev Chil Pediatr 2009; 80 (6): 520-527

\section{Antecedentes}

Los estilos de crianza parentales han sido objeto de creciente interés público y científico en nuestro medio. Diversos estudios chilenos han documentado el aumento de diversas conductas de riesgo juveniles ${ }^{1,2}$ así como la influencia de la familia en su aparición ${ }^{3}$. Interesa conocer entonces la variación de estos estilos de crianza con la edad, para así lograr una mejor definición en el diseño de programas de promoción de la salud y preventivos de riesgo, por el de factores resiliencia de Blum y otros ${ }^{4}$. Estos modelos se adaptaron en los 90 en Chile, utilizando los instrumentos ECRA, CTF y CSF (Escala de Conductas de Riesgo Adolescentes- ${ }^{5}$, Cómo es Tu Familia y Cómo es Su Familia- ${ }^{6}$ para cuantificar esta interacción. En la Universidad de Harvard, Stuart Hauser ${ }^{7}$, mostró como las familias habilitadoras y restrictivas pueden influir en la frecuencia de estas conductas. Posteriormente, Brian Barber en la Universidad de Tennessee ${ }^{8}$, planteó un modelo interaccional, que luego validó en un estudio transcultural, diseñando la escala CNAP (Cross National Adolescent Program) y describiendo tres dimensiones de estilos de parentalidad, encontrando un componente de apoyo y aceptación y otro de control definido por conductas controladoras y disciplinarias. Reitera las tres dimensiones identificadas ya en 1965 por Schaefer ${ }^{9,10}$ de "aceptación vs rechazo", "control vs autonomía psicológica" y "control firme vs control laxo". La primera alude a la aceptación emocional del niño por parte de los padres, y las segundas a las interacciones utilizadas por los padres para controlar a sus hijos. Barber llama a las últimas control psicológico y conductual, las que considera como un factor diferenciador en el comportamiento juvenil: "aceptación-rechazo" tiene un polo positivo caracterizado por compartir experiencias, expresar afecto hacia los hijos, y uno negativo caracterizado por desinterés, negligencia y rechazo. En tanto, la dimensión "Control Psicológico" se refiere a un tipo de control parental que interfiere en el desarrollo psicológico y emocional del niño (ej. invalidación de sentimientos, restricción de expresiones verbales, retiro de amor e inducción de culpabilidad). Finalmente, la dimensión "Control conductual" se centra en "control firme $v s$ control laxo" y se define por "dejar hacer" con autonomía extrema en un polo y castigo y estrictez en el otro polo. Esta dimensión indica el grado en que los padres establecen reglas y regulaciones y las hacen cumplir, poniendo límites a las actividades del adolescente.

Algunos de los resultados relevantes de la 
aplicación transcultural de Barber y cols ${ }^{11}$, señalan que:

- las conductas de aceptación parental estarían relacionadas positivamente con el desarrollo de competencia interpersonal del adolescente, y el tipo de interacciones con sus pares y adultos;

- el control psicológico de los padres tendría una asociación positiva con mayores niveles de síntomas depresivos en los adolescentes;

- el control conductual de los padres estaría negativamente relacionado a las conductas antisociales de los adolescentes, sea facilitando la autorregulación necesaria para que ellos inhiban conductas trasgresoras y se involucren en aquellas socialmente aprobadas, o bien que aquellos adolescentes que carecen de supervisión parental tienen más probabilidad de ser influenciados por sus pares, quienes podrían fomentar conductas riesgosas y desviadas. Las correlaciones encontradas por Barber en su estudio recién mencionado se muestran en la figura 1 .

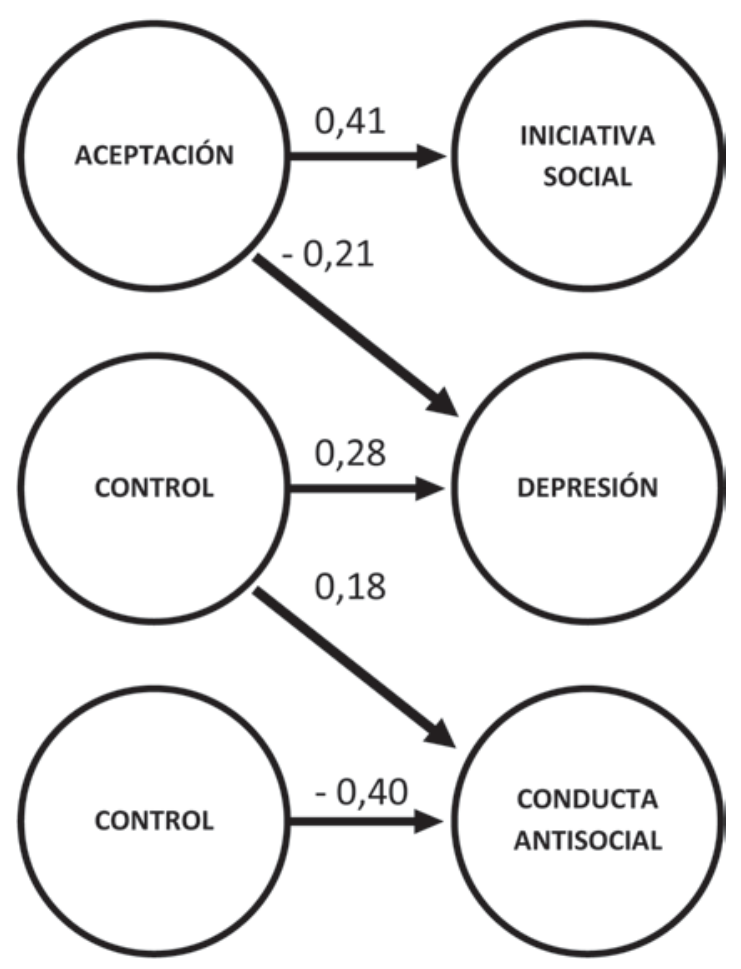

Figura 1. Correlaciones entre estilos de crianza parental y conducta adolescente (Modificado de Barber, 2005).
Este modelo ha sido adaptado en Chile por los autores, en conjunto con Barber ${ }^{11}$, teniendo como referencia que otros estudios nacionales han encontrado evidencia coherente con este modelo. Entre ellos está el del Consejo Nacional de Control de Estupefacientes (CONACE, 2007) que muestra que el grado de involucramiento que tienen los cuidadores con sus hijos, son un factor protector importante ante las conductas de riesgo. En ese contexto, los autores han intentado corroborar en nuestro medio la existencia de asociaciones entre la conducta aceptadora parental y la manifestación de iniciativa social en los adolescentes, entre el control psicológico parental y los síntomas depresivos, entre el bajo control conductual de los padres y las manifestaciones de conductas antisociales en los hijos.

Sin embargo, no se ha explorado sistemáticamente la relación entre la variación de dichas conductas en relación a la edad: la evidencia epidemiológica internacional y chilena muestran que las conductas depresivas aumentan en la medida que el adolescente avanza en su desarrollo personal, en tanto que las conductas antisociales disminuyen. El objetivo de este estudio es aportar evidencia de que dichas variaciones pudieran explicarse por el tipo de control que los padres ejercen en la medida que sus hijos maduran.

\section{Pacientes y Métodos}

Se aplica una adaptación chilena ("Cuestionario para Jóvenes) a partir la encuesta CNAP Plus antes citada. Esta adaptación se preparó en sucesivas reuniones en las que participó el Dr Barber: primero en Bellaggio, Italia (2006), y luego en Concepción, Chile (2006 y 2007). Esta primera aplicación chilena fue realizada el año 2007 en una muestra aleatoria trietápica (por comuna, establecimiento y curso) de estudiantes pertenecientes a 14 establecimientos educacionales de enseñanza media de la Región Metropolitana, seleccionados a partir del listado de establecimientos educacionales del Ministerio de Educación, sorteando comunas estratificadas por nivel socio-económico, luego establecimientos municipales, particulares sub- 
vencionados y privados, y finalmente cursos, llegando a un total de 1447 adolescentes, por lo que todos los colegios tenían la misma probabilidad de ser escogidos. No se consideraron los colegios o escuelas de educación especial para discapacitados.

La encuesta se aplicó previa autorización de los establecimientos, por encuestadores entrenados que visitaron los colegios para la administración presencial de la prueba. El instrumento cuenta con las escalas de depresión, comportamiento antisocial y agresividad de Achenbach; escalas de aceptación parental y control psicológico de Barber, una escala de control conductual de Brown y otra escala de iniciativa social de Bachman y Barber. Se han realizado paralelamente aplicaciones piloto en Bolivia, México y España.

Para estudiar las diferencias según el grupo de edad (bajo y sobre los 15 años) se segmentó la base de datos entre adolescentes menores de 15 años (pre-adolescentes) y adolescentes sobre 15 años (adolescentes medios). Se establecen los 15 años de edad como punto de corte, dado que a esa edad en el sistema chileno de salud los adolescentes pasan de ser vistos desde Pediatría y hospitales de niños, a Medicina de adultos y a hospitales generales.

Se describen las características generales de la muestra mediante estadísticos descriptivos, y se explora el modelo propuesto por Barber mediante correlaciones de Pearson, que correlaciona las variables de percepción de estilos de parentalidad (Apoyo Social, Control Psicológi- co y Control Conductual) con las variables de funcionamiento adolescente (Depresión, Comportamiento Antisocial e Iniciativa Social). Además se establecen diferencias tanto en las variables del modelo (prueba $t$ para muestras independientes) como para los ítems dentro de las variables con diferencias significativas (U de Mann Whitney), entre los 2 grupos etáreos.

\section{Resultados}

De un total de 1447 adolescentes encuestados, 1361 registran datos referentes a edad y sexo, encontrándose un total de $41,7 \%$ de hombres y un 58,3\% de mujeres en la totalidad de la muestra. La tabla 1 muestra la distribución por sexo en cada grupo etario.

Las correlaciones negativas entre aceptación parental y depresión se muestran como las más fuertes en los dos grupos de edad: $-0,438$ $(\mathrm{p}=0,001)$ para los adolescentes bajo 15 años y de $-0,407(p=0,001)$ en el grupo mayor. Por otro lado, la depresión se relaciona positiva y significativamente con el control psicológico parental: $0,313(\mathrm{p}=0,001)$ en el grupo de menor edad y con un 0,366 en el grupo de adolescentes mayores. La tabla 2 ilustra la correlación negativa entre aceptación parental y depresión, en ambos grupos de edad. Estos datos muestran que la acogida emocional de los padres disminuye la posibilidad de que el hijo se sienta depresivo, y que por el contrario el control emocional aumenta dicha posibilidad. Esto su-

Tabla 1. Distribución comparativa por edad y sexo entre adolescentes encuestados en la Región Metropolitana, 2007 ( $n=1422$ )

\begin{tabular}{|c|c|c|c|c|c|}
\hline Género & & & Frecuencia & Porcentaje & Porcentaje válido \\
\hline \multirow[t]{5}{*}{ Masculino } & Válidos & Menor de 15 años & 220 & 37,1 & 39,5 \\
\hline & & Mayor de 15 años & 337 & 56,8 & 60,5 \\
\hline & & Total & 557 & 93,9 & 100,0 \\
\hline & Perdidos & & 36 & 6,1 & \\
\hline & Total & & 593 & 100,0 & \\
\hline \multirow[t]{5}{*}{ Femenino } & Válidos & Menor de 15 años & 356 & 42,9 & 44,3 \\
\hline & & Mayor de 15 años & 448 & 54,0 & 55,7 \\
\hline & & Total & 804 & 97,0 & 100,0 \\
\hline & Perdidos & & 25 & 3,0 & \\
\hline & Total & & 829 & 100,0 & \\
\hline
\end{tabular}


cede tanto para adolescentes mayores como menores.

En cuanto a comportamiento antisocial, se encuentran diferencias estadísticamente significativas $(p=0,001)$ : el grupo de mayor edad presenta más comportamientos antisociales (tabla 3), asi como tiende a consumir más alcohol y drogas. Esta es la única variable que presenta diferencias entre ambos grupos. Los itemes que miden los atributos de comportamiento antisocial en algunos casos apuntan a comportamiento agresivos ("me involucro en riñas de pandillas", "amenazo con golpear a los demás", etc) y otros tienden al consumo de alcohol y drogas. Las diferencias estadísticamente significativas encontradas corresponden al consumo, y no a los comportamientos agresivos (tabla 4).

Tabla 2. Correlaciones de Pearson entre dimensiones del modelo de crianza de Barber et al y depresión variables de funcionamiento adolescente, por grupo de edad en adolescentes encuestados en Región Metropolitana $(n=1447)$

\begin{tabular}{|c|c|c|c|c|c|}
\hline Edad & Variable & & $\begin{array}{c}\text { Control } \\
\text { psicológico } \\
\text { parental }\end{array}$ & $\begin{array}{c}\text { Control } \\
\text { conductual } \\
\text { parental }\end{array}$ & $\begin{array}{c}\text { Apoyo } \\
\text { social } \\
\text { parental }\end{array}$ \\
\hline \multirow[t]{9}{*}{ Menor de 15 años } & Depresión & R de Pearson &, $313(* *)$ &,$- 269(* *)$ &,$- 438(* *)$ \\
\hline & & Sig. (bilateral) &, 000 &, 000 &, 000 \\
\hline & & $\mathrm{N}$ & 466 & 508 & 461 \\
\hline & Comportamiento antisocial & R de Pearson &, $270(* *)$ &,$- 270(* *)$ &,$- 203(* *)$ \\
\hline & & Sig. (bilateral) &, 000 &, 000 &, 000 \\
\hline & & $\mathrm{N}$ & 457 & 493 & 452 \\
\hline & Iniciativa social & R de Pearson &,- 032 &, 080 &, $\left.1922^{* *}\right)$ \\
\hline & & Sig. (bilateral) & ,494 &, 069 &, 000 \\
\hline & & $\mathrm{N}$ & 473 & 515 & 469 \\
\hline \multirow[t]{9}{*}{ Mayor de 15 años } & Depresión & R de Pearson &, $366(* *)$ &,$- 264(* *)$ &,$- 407(* *)$ \\
\hline & & Sig. (bilateral) &, 000 &, 000 &, 000 \\
\hline & & $\mathrm{N}$ & 612 & 675 & 604 \\
\hline & Comportamiento antisocial & R de Pearson &, $286\left(^{* *}\right)$ &,$- 324(* *)$ &,$- 270\left(^{* *}\right)$ \\
\hline & & Sig. (bilateral) &, 000 &, 000 &, 000 \\
\hline & & $\mathrm{N}$ & 609 & 669 & 601 \\
\hline & Iniciativa social & R de Pearson &,$- 125(* *)$ &, $135(* *)$ &, $247\left(^{* *}\right)$ \\
\hline & & Sig. (bilateral) &, 002 &, 000 &, 000 \\
\hline & & $\mathrm{N}$ & 615 & 677 & 605 \\
\hline
\end{tabular}

*Rxy: coeficiente de Correlación de Pearson. **La correlación es significativa al nivel 0,01 (bilateral).

Tabla 3. Diferencia de medias (prueba t) en los puntajes de escala de comportamiento antisocial, comparados por edad en adolescentes encuestados en la Región Metropolitana (2007), prueba de Levene para muestras independientes

\begin{tabular}{|c|c|c|c|c|c|c|c|c|c|}
\hline & \multicolumn{2}{|c|}{ Levene* } & \multicolumn{7}{|c|}{ Prueba T para la igualdad de medias } \\
\hline & \multirow[t]{2}{*}{$\mathbf{F}$} & \multirow[t]{2}{*}{ Sig. } & \multirow[t]{2}{*}{$\mathbf{t}$} & \multirow{2}{*}{ GI } & \multirow{2}{*}{$\begin{array}{c}\text { Sig. } \\
\text { (bilateral) }\end{array}$} & \multirow{2}{*}{$\begin{array}{c}\text { Diferencia } \\
\text { de } \\
\text { medias }\end{array}$} & \multirow{2}{*}{$\begin{array}{l}\text { Error típ. } \\
\text { de la } \\
\text { diferencia }\end{array}$} & \multicolumn{2}{|c|}{ Intervalo } \\
\hline & & & & & & & & Superior & Inferior \\
\hline Comporta & & & & & & & & & \\
\hline antisocial & 6,723 & 01 & $-3,949$ & 1234,147 &, 000 &,- 54218 &, 13730 &,- 81155 &,- 27281 \\
\hline
\end{tabular}

*Prueba de Levene para la igualdad de varianzas. 
Tabla 4. Comparación por edad en nivel de consumo de sustancias químicas mediante U de Mann Whitney (adolescentes sobre y bajo 15 años encuestados en la Región Metropolitana de Santiago $(n=1447)$

\begin{tabular}{lcrrr}
\hline & $\begin{array}{c}\text { Tomas alcohol, } \\
\text { cerveza, vino o } \\
\text { tragos fuertes }\end{array}$ & Usas marihuana & $\begin{array}{c}\text { Usas drogas duras } \\
\text { (pasta base, heroina, } \\
\text { cocaina y LSD) }\end{array}$ & Fumas cigarrillos \\
\hline U de Mann-Whitney & 154940 & 207372 & 220350 & 176377 \\
W de Wilcoxon & 319391 & 372972 & 385375 & 341402 \\
Z & -10359 & -4323 & -2148 & -7805 \\
Sig. asintót. (bilateral) & 0 & 0 & 032 & 0 \\
\hline
\end{tabular}

De modo que al comparar a estudiantes de menos de 15 años con los de más de 16 y en forma significativa, los menores presentan:

- Menor consumo frecuente de alcohol (semanal a diario): $16 \%$ en comparación a un $38 \%$ de los mayores $\left(\chi^{2}=107,95, \mathrm{p}=0,0001\right)$.

- Fuman menos tabaco con frecuencia un $19,9 \%$ de los menores en comparación a un $38,4 \%$ de los mayores $\left(\chi^{2}=63,02, p=0,0001\right)$.

- Consumen marihuana en alguna cantidad un $7,7 \%$ de los menores en comparación a un $15,3 \%$ de los mayores $\left(\chi^{2}=18,78, p=0,001\right)$;

- Consumen cocaína u otras drogas duras un $1,4 \%$ de los menores en comparación a un $3,3 \%$ de los mayores $\left(\chi^{2}=8,995, p=0,06\right)$.

Cabe señalar que no hubo diferencias estadísticamente significativas por edad en la presencia de riñas frecuentes, sean individuales o grupales, conductas vandálicas, robos menores o historia de arrancarse de la casa. Tampoco hay diferencias por grupo etario en la presencia de ideación suicida o de síntomas depresivos.

Al comparar por género se encontraron diferencias significativas:

- En el consumo de sustancias químicas, con mayor uso frecuente de alcohol entre los hombres $(33,3 \%$ vs $22,2 \%$ entre las mujeres) $\left(\chi^{2}=25,53, p=0,0001\right)$.

- En el consumo de marihuana (17,3\% de los hombres vs $9,9 \%$ de las mujeres $)\left(\chi^{2}=32,888\right.$, $\mathrm{p}=0,0001)$ y de cocaína $\mathrm{u}$ otras drogas $(5,3 \%$ de los hombres vs $1,3 \%$ de las mujeres) $\left(\chi^{2}=21,439, p=0,0001\right)$.

En cambio, no hubo diferencias por género en el uso de tabaco, en tanto sí se identifican en las conductas hetero-agresivas:
- Robos menores $(12,2 \%$ de los hombres vs $5,5 \%$ de las mujeres $)\left(\chi^{2}=31,749, \mathrm{p}=0,0001\right)$;

- En las riñas en el colegio (20,2\% de los hombres vs $10,1 \%$ de las mujeres $)\left(\chi^{2}=39,037\right.$, $\mathrm{p}=0,0001)$

- En las peleas en pandillas $(16,1 \%$ de los hombres $v s 4 \%$ de las mujeres $)\left(\chi^{2}=62,476\right.$, $\mathrm{p}=0,0001)$. Por otra parte, informan haber presentado tristeza o depresión moderadas a severas un $30,6 \%$ de los hombres $v s$ un $39 \%$ de las mujeres $\left(\chi^{2}=63,02, p=0,004\right)$; han pensado frecuentemente en matarse un $6 \%$ de los hombres $v s$ un $8,9 \%$ de las mujeres $\left(\chi^{2}=9,725, p=0,008\right)$.

\section{Discusión}

Este estudio corrobora y valida en Chile el modelo explicativo de conducta adolescente desarrollado por Barber y cols, a partir de la percepción que los jóvenes tienen de las relaciones con sus padres, como lo ilustran las figuras 1 y 2 , que comparan las correlaciones encontradas originalmente por Barber con las chilenas. Tanto en la muestra internacional como en la chilena, a mayor aceptación parental, hay mayor iniciativa social y menor depresión por parte de los jóvenes. Esto corrobora la importancia de la aceptación emocional del padre hacia el hijo, como elemento que fomenta un desarrollo infanto juvenil adecuado. La no aceptación y el control psicológico se correlacionan con más depresión y más conductas antisociales y el control conductual se correlaciona con menos comportamientos antisociales. Esto va en línea con las investigaciones que muestran que los padres que controlan psicológicamente 
tienen hijos con mayores dificultades de autoestima, y que los que no controlan conductualmente tienen hijos con mayores problemas del comprortamiento. Por otra parte, este estudio diferencia parcialmente la variabilidad a lo largo del desarrollo adolescente dicotomizado por grupos etarios menores o mayores de quince años (figura 2).

El modelo empleado propone que a mayor aceptación parental, hay mayor iniciativa social. Esto es corroborado por este estudio, que encuentra una diferencia significativa especialmente en el grupo de mayor edad comparado con el de menor edad. Esto resulta de interés, dado que a medida que el joven crece, aumentan sus interacciones sociales: estos datos apuntan a que el apoyo emocional parental promueve una creciente exploración del mundo. Esto confirma la afirmación de psicólogos evolutivos y autores psicoanalíticos como Erik Erikson ${ }^{12}$, que muestran que la mejor manera de que el niño se abra al mundo es que tenga una confianza básica en la actitud positiva de sus padres. Además, la falta de aceptación parental se correlaciona con sintomatología depresiva en el adolescente: si éste no percibe apoyo consistente de parte de sus padres tiene más ánimo depresivo. Esta correlación es algo mayor en el grupo de menor edad, que cuenta con menos espacios de relación fuera de la familia que el grupo de mayores de 15 años.

Los jóvenes que perciben un mayor control psicológico de parte de sus padres, tienen una mayor tendencia a las conductas antisociales y a la depresión. Entre las dimensiones de la escala de control psicológico está la de manipulación a través de la culpa que ejercen las figuras de cuidado, por ejemplo, "Es menos amistosa/o conmigo cuando no veo las cosas a su manera. Evita mirarme cuando le desilusiono, si hiero sus sentimientos, deja de hablarme hasta que la/o agrado de nuevo", "me culpa por problemas de otros miembros de la familia”. La conducta antisocial que presenta mayores diferencias es la del consumo de sustancias que se puede considerar una conducta autoagresiva más que heteroagresiva. Este aspecto podría ser explorado con mayor profundidad, con metodologías cualitativas.

Finalmente, se encuentra que el control conductual parental, ejercido como fijación de límites claros y bien definidos, además de un monitoreo de lo que los adolescentes hacen es

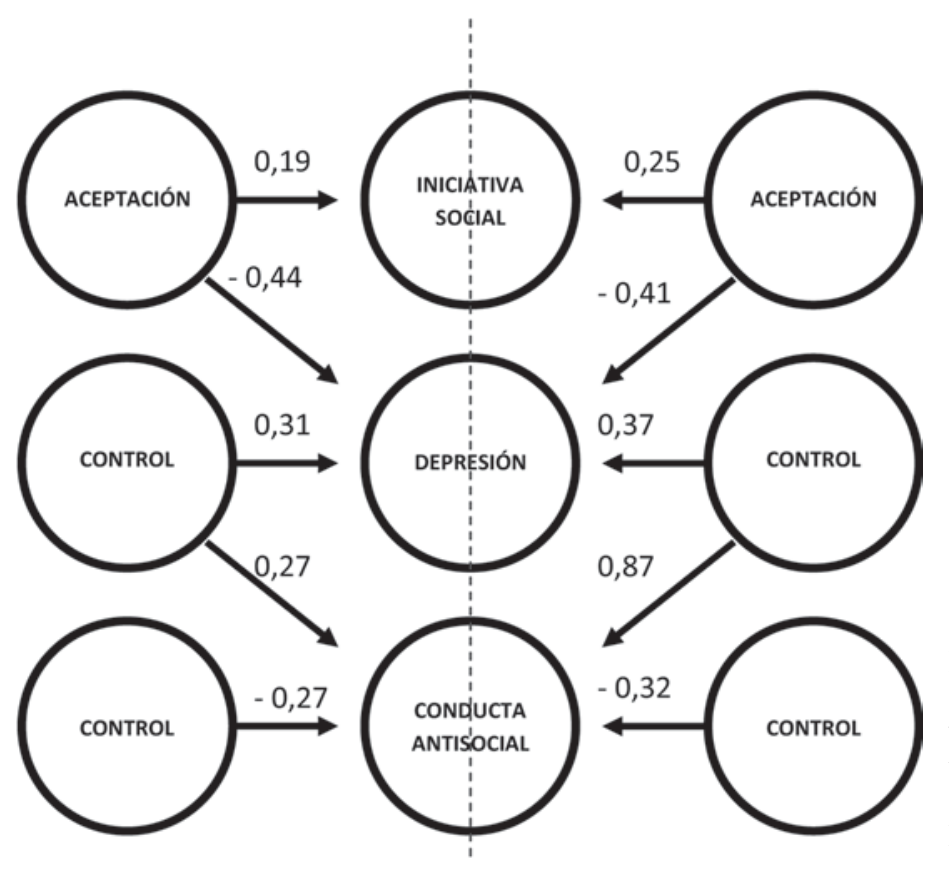

Figura 2. Correlaciones de las dimensiones de aceptación parental, control psicológico y conductual con variables juveniles (iniciativa social, depresión y conducta antisocial) en jóvenes sobre y bajo 15 años, Región Metropolitana, Chile, 2007. 
un factor protector del comportamiento antisocial. De acuerdo con esto, el desafío de los padres sería el establecer límites claros, sin caer en una percepción de hostigamiento, que como ya se indicó tendría un efecto contrario.

Estos resultados tienen claras implicancias para programas de intervención con jóvenes, al acentuar la importancia de sentirse acogidos y aceptados por los padres, o en ausencia de éstos, por adultos que establezcan relaciones de cuidado con ellos. Asimismo, diferencia el control psicológico, con su efecto a veces culpabilizante, del conductual, mostrando la mayor eficacia del último en promover comportamientos pro-sociales.

En trabajos futuros es necesario determinar cómo opera el modelo desde una perspectiva de género, dadas las diferencias encontradas acerca de como perciben hombres y mujeres el control conductual, psicológico y la aceptación parental. Asimismo, es importante correlacionar dicha percepción hacia el padre y la madre por separado.

Parece importante la difusión de estos resultados pues su manejo por instancias estatales y privadas puede condicionar el diseño e implementación de programas que fortalezcan la relación "parentalidad-filiación", en la perspectiva de un adecuado funcionamiento familiar que, efectivamente, se caracterice por generar ambientes protectores que prevengan conductas que afectan el entorno social.

\section{Referencias}

1.- CONACE: Séptimo Estudio Nacional de Drogas en Población General de Chile. Ministerio del Interior, Chile, 2006.
2.- Florenzano $U R$, Valdés $C M$ : El adolescente y sus conductas de riesgo. Ediciones Universidad Católica de Chile, Santiago de Chile. 2005.

3.- Santander S, Zubarew T, Santelices L, Argollo A, Cerda J, Borquez M: Influencia de la familia como factor protector de conductas de riesgo en escolares chilenos. Rev Méd Chile, 2008: 136: 317-24.

4.- Blum $R$ : The health of young people in a global context. J Adolesc Health 2004; 35: 402-18.

5.- Marchandón A, Florenzano R, Pino P: Adaptación en Chile de un cuestionario para medir conductas de riesgo en adolescentes. Actas del II Encuentro de Investigadores Médico-Sociales sobre la Juventud, Santiago de Chile, 1992.

6.- Hernández A, Beuhring T, Berner E, et al: Familia y Adolescencia: Indicadores de Salud. Manual de aplicación de Instrumentos. Organización Panamericana de la Salud, Washington D.C. 1996.

7.- Hauser ST, Powers S, Noam G: Adolescents and Their Families: Paths of Ego Development. New York: Free Press, 1991.

8.- Barber $B K$ : Intrusive parenting: How psychological control affects children and adolescents. Washington, DC: American Psychological Association Press. 2002.

9.- Schaefer ES: Children's reports of parental behavior: An inventory. Child Development 1965a; 36: 41324.

10.- Schaefer ES: A configurational analysis of children's reports of parent behavior. Journal of Consulting Psychology 1965b; 29: 552-7.

11.- Florenzano $R$, Cáceres $E$, Valdés $M$, Santander $S$, Calderón S, Casassus $M$, et al: Conductas de riesgo y factores de protección en una muestra representativa de adolescentes escolarizados en la Región Metropolitana. Actas del III Congreso Chileno de Adolescencia, Santiago de Chile 4 al 6 de Septiembre de 2008.

12.- Erikson E: Infancia y Sociedad. Buenos Aires, Hormé, 1964. 